\title{
Atypical perinuclear antineutrophil cytoplasmic antibodies after colectomy in inflammatory bowel disease
}

\author{
Hugh J Freeman MD, Brenda Roeck RT, Dana V Devine PhD, Cedric J Carter MD
}

\begin{abstract}
HJ Freeman, B Roeck, DV Devine, CJ Carter. Atypical perinuclear antineutrophil cytoplasmic antibodies after colectomy in inflammatory bowel disease. Can J Gastroenterol 1997;11(4): 305-311. Atypical perinuclear antineutrophil cytoplasmic antibodies (p-ANCA) have been detected in most patients with ulcerative colitis and primary sclerosing cholangitis. Persistent atypical p-ANCA have been observed in ulcerative colitis patients with a prior proctocolectomy, especially with pouchitis, suggesting that this serological marker might be predictive of subsequent development of chronic or refractory pouchitis. This study prospectively evaluated this serological marker in 24 consecutive patients with inflammatory bowel disease and prior colectomies (12 with a clinical diagnosis of ulcerative colitis and 12 with a clinical diagnosis of Crohn's disease involving the colon). Of these, 14 were positive, including 11 with extensive ulcerative colitis and three with Crohn's disease. Although two of three ulcerative colitis patients with pouchitis were positive, eight of eight ulcerative colitis patients having a pelvic pouch with no pouchitis were also positive, as was a patient who elected to have an end-ileostomy (Brooke's ileostomy). Two patients had abnormal liver chemistry tests. Both had end-stage primary sclerosing
\end{abstract}

cholangitis treated with liver transplantation and were positive for this serological marker. Although atypical p-ANCA may be a marker of persistent inflammation in pelvic pouch patients, a positive test result should not be used for prognosis or as a decision-making parameter for pelvic pouch procedures.

Key Words: Antineutrophil cytoplasmic antibodies, Continent ileostomy, Crohn's disease, Inflammatory bowel disease, Ulcerative colitis

\section{Anticorps cytoplasmiques antineutro- philes périnucléaires atypiques après colectomie dans la maladie inflammatoire de l'intestin}

RÉSUMÉ : Des anticorps cytoplasmiques antineutrophiles atypiques périnucléaires ( $\mathrm{p}$-ANCA) ont été décelés chez la plupart des patients souffrant de colite ulcéreuse et de cholangite sclérosante primitive. La présence de p-ANCA persistants atypiques a été observée dans la colite ulcéreuse chez les patients

Pour le résumé, voir page suivante

Departments of Medicine (Gastroenterology) and Laboratory Medicine (Special Hematology), University of British Columbia, Vancouver, British Columbia

Correspondence and reprints: Dr Hugh Freeman, ACU F-137, Gastroenterology, University Hospital, 2211 Wesbrook Mall, Vancouver, British Columbia V6T 1W5. Telephone 604-822-7216, fax 604-822-7236

Received for publication August 25, 1996. Accepted November 15, 1996 
ayant déjà subi une proctocolectomie, surtout avec pouchite, ce qui donne à penser qu'il s'agirait d'un marqueur sérologique permettant de prédire l'installation de pouchite «chronique» ou «réfractaire». Cette étude visait à évaluer de façon prospective ce marqueur sérologique chez 24 patients consécutifs atteints de maladie inflammatoire de l'intestin et ayant déjà subi des colectomies ( 12 cas cliniques de colite ulcéreuse et 12 cas cliniques de maladie de Crohn avec atteinte colonique). Parmi ces sujets, 14 étaient positifs, dont 11 présentaient une colite ulcéreuse étendue et trois une maladie de Crohn. Bien que deux des trois patients atteints de colite ulcéreuse présentant une pouchite se soient révélés positifs, les huit patients ayant une colite ulcéreuse avaient un réservoir pelvien sans pouchite et étaient également positifs, comme l'était un patient qui a choisi de subir une iléectomie de Brooke. Deux patients présentaient des résultats anormaux à leurs tests de biochimie hépatique. Les deux présentaient une cholangite sclérosante primitive terminale avec transplantation hépatique et étaient positifs à l'égard de ces marqueurs sérologiques. Bien que la présence de p-ANCA atypique puisse être un marqueur de l'inflammation persistante chez les patients présentant un réservoir pelvien, la présence de résultats positifs au test ne devrait pas être utilisée pour le pronostic, mais comme paramètre décisionnel pour des interventions ayant trait aux réservoir pelvien.
A typical perinuclear antineutrophil cytoplasmic antibodies ( $\mathrm{p}$-ANCA) have been recognized in most patients with idiopathic ulcerative colitis and primary sclerosing cholangitis $(1,2)$. In a recent prospective Canadian study of 500 consecutive patients with inflammatory bowel disease from a single clinical practice, $66.3 \%$ of patients with ulcerative colitis and $11.8 \%$ with Crohn's disease were positive for this serological marker (3). In the same study, patients with concomitant primary sclerosing cholangitis also had atypical p-ANCA (3). These results were similar to those reported from other centres $(2,4)$.

Because the target organ of the disease is lost, removal of the colon in ulcerative colitis patients might alter ANCA detection, especially if these antibodies represent an epiphenomenon of active colonic inflammation (5). In limited studies, however, ANCA were still detectable after total colectomy for colitis in up to $60 \%$ of cases (6-9). Moreover, in cases where pouchitis was present, most (7-9), but not all (10), series have reported a very high prevalence of ANCA. Therefore, it has been suggested that ANCA may be used as markers to predict pouchitis following ileo-anal pouch anastomoses $(6,7)$.

This report describes ANCA serological studies in a consecutive series of 24 patients with inflammatory bowel disease treated with colectomy. The goals were to classify these patients serologically after colectomy with a prior clinical diagnosis of either ulcerative colitis or Crohn's disease and to review their clinical course following colonic resection. In two patients, concomitant sclerosing cholangitis was complicated by hepatic failure, encephalopathy and/or stomal variceal hemorrhage followed by orthotopic liver transplantation.

\section{PATIENTS AND METHODS}

Patient groups: Each patient had an established clinical diagnosis of ulcerative colitis or Crohn's disease in the small and/or large intestine, based on clinical, radiological, endoscopic and histologic criteria as well as on negative microbiological studies (11). Pouchitis was diagnosed on the basis of previously established criteria (12). All samples for serological studies were collected in a consecutive fashion with no exclusions or refusals.
Twelve patients had ulcerative colitis. Colonoscopy and histological studies showed a diffuse mucosal inflammatory process in the rectum that extended proximally within the colon. Extensive disease, defined as greater than $60 \mathrm{~cm}$ from the anal verge (3), was present in all 12 patients. All ulcerative colitis patients were offered a colectomy and pelvic Jtype pouch as a staged procedure. Of these patients, one requested a single stage proctocolectomy with an end (Brooke's) ileostomy. One also had concomitant primary sclerosing cholangitis and developed hepatic failure. Hepatic transplantation was done at another hospital, but the patient subsequently died. Since colectomy, the other 11 patients have not received steroids or immunosuppressant medications.

The Crohn's disease group also comprised 12 patients. Colonoscopy and histological studies showed segmental or patchy inflammatory changes within the colorectum and/or distal small intestine, mostly with histological features of a granulomatous inflammatory process. In addition, endoscopic mucosal biopsies and/or radiological evaluations of the upper gastrointestinal tract were done. Surgical materials were also reviewed.

Six of the 12 patients with Crohn's colitis also had small intestinal disease requiring resection. Of these, two underwent repeated small intestinal resections that resulted in a continuous requirement for parenteral nutrition.

Of the 12 patients, 11 had granulomatous inflammation confirmed by histological evaluation of endoscopic biopsies or surgical specimens of the colon and/or small intestine. Only one patient had no demonstrable multinucleated giant cells or granulomas. In one patient with Crohn's disease of the colon and distal ileum with associated granulomatous inflammation in the excised intestine, a diagnosis of primary sclerosing cholangitis was defined later in his clinical course, which became complicated by portal hypertension and bleeding ileostomy varices. Because of these complications, liver transplantation was done at another hospital. This patient is currently maintained on prednisone and cyclosporine.

Two of the reported patients were initially diagnosed with Crohn's colitis in the hospital. One had granulomas in two rectal biopsies. Patients were referred elsewhere by family 
TABLE 1

Inflammatory bowel disease patients with prior proctocolectomy

\begin{tabular}{|c|c|c|c|c|c|c|}
\hline Patient & Age & Sex & Clinical diagnosis & ANCA & Granulomas & Current treatment \\
\hline 1 & 20 & Female & $C D$ & - & + & Ileostomy \\
\hline 2 & 22 & Male & $C D$ & - & + & Ileostomy \\
\hline 3 & 20 & Male & UC & + & - & Pelvic pouch \\
\hline 4 & 22 & Female & $C D$ & - & + & Ileostomy \\
\hline 5 & 14 & Male & $C D$ & - & + & Ileostomy and recurrence \\
\hline $6^{*}$ & 28 & Female & UC & + & - & Pelvic pouch \\
\hline $7^{\dagger}$ & 25 & Female & $C D$ & - & + & Ileostomy \\
\hline 8 & 37 & Male & UC & + & - & Pelvic pouch \\
\hline 9 & 17 & Female & UC & + & - & Pelvic pouch and pouchitis \\
\hline 10 & 21 & Female & CD & - & + & Ileostomy and recurrence \\
\hline 11 & 26 & Female & $C D$ & - & + & Ileostomy \\
\hline $12^{\dagger}$ & 11 & Female & $C D$ & - & + & lleostomy \\
\hline 13 & 26 & Female & $C D$ & - & + & Ileostomy \\
\hline 14 & 26 & Female & UC & + & - & Pelvic pouch \\
\hline $15^{*}$ & 14 & Male & $C D$ & + & + & Ileostomy \\
\hline 16 & 39 & Male & UC & + & - & Pelvic pouch \\
\hline 17 & 30 & Male & UC & + & - & Pelvic pouch and pouchitis \\
\hline 18 & 17 & Female & CD & + & - & Pelvic pouch and pouchitis \\
\hline 19 & 27 & Male & UC & - & - & Pelvic pouch and pouchitis \\
\hline 20 & 32 & Male & UC & + & - & Pelvic pouch \\
\hline 21 & 22 & Female & $C D$ & + & + & Pelvic pouch, pouchitis and fistula \\
\hline 22 & 10 & Female & UC & + & - & Ileostomy \\
\hline 23 & 26 & Male & UC & + & - & Pelvic pouch \\
\hline 24 & 32 & Female & UC & + & - & Pelvic pouch \\
\hline
\end{tabular}

${ }^{*}$ Primary sclerosing cholangitis and subsequent liver transplant; ${ }^{\dagger}$ Receiving total parenteral nutrition from home. ANCA Atypical perinuclear antineutrophil cytoplasmic antibodies; CD Crohn's disease; UC Ulcerative colitis 
physicians for surgical treatment of colitis, which comprised colectomy and creation of a pelvic pouch. In both of these patients, severe pouchitis with focal ulceration in the pouch developed, and in one patient, pouchitis was complicated by an enterovaginal fistula.

Laboratory studies: For each patient, all blood samples were collected into vacutainer glass tubes (Becton Dickinson, New Jersey) by a laboratory technician with no knowledge of the patient's diagnosis or treatment. Blood was also obtained for hematological studies (hemoglobin, white blood cell counts and platelet counts), erythrocyte sedimentation rates, liver chemistry tests (aspartate aminotransferase, alanine aminotransferase and alkaline phosphatase), serum iron studies and serum proteins, including serum albumin. Blood samples were collected into vacutainer glass tubes and allowed to clot at room temperature. The serum was used for ANCA detection by two methods: ANCA indirect immunofluorescence, and, if atypical p-ANCA were detected with this method, then ANCA ELISA was also done. One experienced observer performed immunofluorescence evaluations and was blinded to the clinical diagnosis for each patient.

ANCA immunofluorescence: Indirect immunofluorescence for cytoplasmic ANCA (c-ANCA), p-ANCA or atypical p-ANCA was done as previously reported (3) with a standardized fluorescent antibody detection method using a kit purchased from a commercial supplier (Inova Diagnostics Inc, California). An adherent layer of cultured human neutrophils was applied to each slide. Culture conditions were designed to ensure stability and strong expression of the primary cytoplasmic granules. Adherent neutrophils were fixed by the manufacturer with either ethanol or formalin. The primary screen for c-ANCA or $\mathrm{p}$-ANCA involved incubation of serum at a 1:20 dilution in phosphate buffered saline with ethanol-fixed slides for 25 mins at room temperature followed by a 5 min wash with phosphate buffered saline. This was followed by another $25 \mathrm{~min}$ incubation using affinity purified antihuman immunoglobin $\mathrm{G}(\mathrm{IgG})$ with a fluorescent tag followed by a further $5 \mathrm{~min}$ wash with phosphate buffered saline. Coverslips were applied to the slides, which were examined with a fluorescence microscope at 500x magnification. Positive ANCA cellular fluorescence was recorded and the distribution pattern of fluorescence designated as $\mathrm{c}$ ANCA, $p$-ANCA or atypical p-ANCA. Sera that were positive for atypical $\mathrm{p}$-ANCA were further evaluated using slides fixed with formalin, which destroys nuclear antigens and atypical p-ANCA. In addition, formalin fixes both c-ANCA and P-ANCA antigens in the cytoplasm so that a false positive pattern is not observed.

ELISA assays: ANCA ELISA assays were done as previously reported (3) using a commercial kit (Quanta-Lite MPO or PR3 ELISA, Inova Diagnostics Inc) that uses microtitration strips containing wells coated with proteinase-3, a primary antigen related to the c-ANCA pattern, or myeloperoxidase, a primary antigen associated with the p-ANCA pattern. Diluted serum was applied and incubated. Specific antibodies were bound to the wells. Unbound material was initially removed by washing. Bound antibodies were detected by adding enzyme-labeled antihuman IgG followed by a second washing and incubated with a nitrophenol substrate. Wells with antiproteinase- 3 or antimyeloperoxidase antibodies were quantitated by colorimetry. In this study, the results of ELISA were used to confirm the results of ANCA immunofluorescence.

\section{RESULTS}

ANCA detection rates: Results in patients with inflammatory bowel disease, with or without primary sclerosing cholangitis, have been previously reported (3). In 500 patients with inflammatory bowel disease, $66.3 \%$ of 247 patients with ulcerative colitis and $11.8 \%$ of 253 patients with Crohn's disease had atypical p-ANCA. Various subgroups of patients with inflammatory bowel disease and atypical $\mathrm{p}$ ANCA specifically relevant to the present report were also described in previously (3). These comprised $69.8 \%$ of patients with extensive ulcerative colitis extending more than $60 \mathrm{~cm}$ from the anal verge; $18.8 \%$ of patients with Crohn's disease of the colon alone; and $12.2 \%$ of patients with Crohn's disease involving the colon and small intestine, usually the ileum. In addition, previous studies from the author's hospital of patients without inflammatory bowel disease or infectious or ischemic colitis failed to demonstrate atypical p-ANCA.

Atypical p-ANCA were found in 11 of 12 (92\% patients after colectomy with a clinical diagnosis of extensive ulcerative colitis and in three of $12(25 \%)$ patients with a clinical diagnosis of Crohn's disease and colonic involvement ( $\mathrm{Ta}$ ble 1). Thus, compared with patients with inflammatory bowel disease who did not undergo proctocolectomy, detection rates after colectomy were higher for extensive ulcerative colitis or Crohn's disease involving the colon (both $\mathrm{P} \leq 0.05)$.

Patient descriptive factors: Table 1 shows descriptive data for patients after colectomy. The mean age of patients with ulcerative colitis (27 years) was significantly different from that of patients with Crohn's disease ( 20 years) $(\mathrm{P} \leq 0.05)$. In the ulcerative colitis group, there were seven males and five females, while in the Crohn's disease group, there were nine females and three males. All patients with an initial clinical diagnosis of ulcerative colitis had mucosal inflammatory disease with no granulomas detected in biopsies or resected specimens. In contrast, 11 of 12 patients with Crohn's disease involving the colon had typical histopathological features in endoscopic biopsies or resected specimens, including multinucleated giant cells and granulomas.

Clinical courses following colectomy: All 24 patients had a prior colectomy. In 10 patients with Crohn's disease and in one patient with ulcerative colitis, a Brooke's ileostomy was created. Thirteen patients underwent pelvic pouch recon- 
struction, including 11 patients with an initial clinical diagnosis of extensive ulcerative colitis. In some patients with Crohn's disease of the distal ileum at colectomy, variable lengths of ileum were also resected along with the colon. Two patients also had repeated small bowel resections that led to a requirement for parenteral nutrition. Since the colon resection, two patients have developed macroscopic evidence of recurrent Crohn's disease in the ileostomy mucosa. Of the 13 pelvic pouch patients, five developed pouchitis, including three patients with an initial clinical diagnosis of extensive ulcerative colitis. The number of pouchitis cases in serologically positive and negative patients did not differ.

Liver function and primary sclerosing cholangitis: All 24 patients had liver chemistry tests during their clinical courses and at the time of ANCA evaluation. Liver chemistry tests were normal in 22. Liver chemistry tests were abnormal in two, and radiological studies revealed primary sclerosing cholangitis. Both patients had complicated clinical courses leading to hepatic resection and transplantation. One with extensive ulcerative colitis died with an intact pelvic pouch. The other had Crohn's disease of the small and large bowel, was treated with an ileal resection, total proctocolectomy and end-ileostomy, and has survived for 10 years after hepatic transplantation and remains well on prednisone and cyclosporine. Both patients had atypical p-ANCA following colectomy and hepatic resection with transplantation.

Serological detection of atypical $\mathrm{p}$-ANCA: Table 1 shows patient descriptive data related to atypical p-ANCA. Fourteen of 24 patients were positive for atypical p-ANCA, including two inflammatory bowel disease patients with atypical p-ANCA and concomitant primary sclerosing cholangitis and 12 without concomitant primary sclerosing cholangitis. Mean ages of atypical p-ANCA-positive and -negative patients were 25.0 and 21.4 years, respectively. Seven males and seven females tested positive for atypical p-ANCA, and three males and seven females tested negative. A review of endoscopic and resected specimens revealed that two of 14 patients with atypical p-ANCA had granulomas while nine of 10 with no detectable atypical p-ANCA had granulomas.

\section{DISCUSSION}

We have previously reported, in a study of 500 consecutive patients with inflammatory bowel disease, that most patients with ulcerative colitis, but not Crohn's disease, were positive for the subclinical serological marker, atypical p-ANCA (3). This finding was consistent with results reported by other investigators with smaller numbers of patients (1).

\section{REFERENCES}

1. Saxon A, Shanahan F, Landers C, Ganz T, Targan S. A distinct subset of antineutrophil cytoplasmic antibodies is associated with inflammatory bowel disease. J Allergy Clin Immunol 1990;86:202-10.
The present report extends our initial findings to a group of 24 patients with prior colectomies for inflammatory bowel disease - either ulcerative colitis or Crohn's disease. Except for two patients with associated primary sclerosing cholangitis who underwent prior colectomy as well as liver transplantation, other extra intestinal features of inflammatory bowel disease were not present. Fourteen of 24 patients with a prior colectomy for intractable colitis were ANCApositive (11 with ulcerative colitis and three with Crohn's disease). Liver chemistry tests were normal in all patients except two with sclerosing cholangitis who were treated with liver transplantation. While it is not clear whether ANCA are involved in the immunopathogenesis of these disorders and possibly reflect a specific immunoregulatory defect, the studies reported here appear to confirm the previous suggestion (5) that ANCA are not simply immunological epiphenoma related to the presence of the target organ and persistent colitis.

In this study of colitis patients, we were able to extend observations to those with superimposed sclerosing cholangitis treated with orthotopic liver transplantation. In this procedure, the entire biliary tree is resected except for the most distal portion of the choledochal duct. As in two of our patients, the detection of atypical p-ANCA has been previously reported after both colectomy and orthotopic liver transplantation $(13,14)$. Because p-ANCA-positive sera do not react with biliary ductal epithelium in hepatic sections (4), these studies suggest that the production of atypical pANCA is independent of diseased colon or liver. In addition, although extensive colitis was well documented in our study group, only two patients had concomitant primary sclerosing cholangitis, a specific factor previously reported to correlate with an increased rate of pouchitis after ileal pouch-anal anastomosis $(15,16)$. Our results contrast sharply with the results of some $(7,8)$, but not all (9), reports and suggest that atypical p-ANCA should not be used as serological markers to predict poor prognoses in colitis patients following colectomy (ie, the development of 'chronic' or 'refractory' pouchitis).

In conclusion, despite the limited patient numbers in this prospective study, atypical p-ANCA appear to persist in a high proportion of patients following colectomy and ileal pouch-anal anastomosis for extensive ulcerative colitis as well as in those who have undergone hepatic transplantation for concomitant sclerosing cholangitis. The presence of atypical $\mathrm{p}$-ANCA in this setting could simply be a marker or epiphenomena of persisting inflamed or diseased rectal tissue, especially with stapled anastomoses or, possibly, inflamed or diseased distal biliary ductal tissue that could not be resected. As such, this serological biomarker should not be used as a decision-making parameter for pelvic pouch procedures in patients with inflammatory bowel disease.

2. Duerr RH, Targan SR, Landers CJ, et al. Neutrophil cytoplasmic antibodies: a link between primary sclerosing cholangitis and ulcerative colitis. Gastroenterology 1991;100:1385-91.

3. Freeman HJ, Roeck B, Devine D, Carter C. Prospective evaluation of 
neutrophil auto-antibodies in 500 consecutive patients with inflammatory bowel disease. Can J Gastroenterol 1997;11:203-7.

4. Seibold F, Weber P, Klein R, Berg PA, Wiedmann KH. Clinical significance of antibodies against neutrophils in patients with inflammatory bowel disease and primary sclerosing cholangitis. Gut 1992;33:657-62.

5. Duerr RH, Targan SR, Landers CJ, Sutherland LR, Shanahan F. Antineutrophil cytoplasmic antibodies in ulcerative colitis. Comparison with other colitides/diarrheal diseases. Gastroenterology 1991;100:1590-6.

6. Patel RT, Stokes R, Birch D, Ibbotson J, Keighley MRB. Influence of total colectomy on serum antineutrophil cytoplasmic antibodies in inflammatory bowel disease. Br J Surg 1994;81:724-6.

7. Sandborn WJ, Landers CJ, Tremaine WJ, Targan SR. Antineutrophil cytoplasmic antibody correlates with chronic pouchitis after ileal pouch-anal anastomosis. Am J Gastroenterol 1995;90:740-7.

8. Aisenberg J, Wagreich J, Shim J, et al. Perinuclear anti-neutrophil cytoplasmic antibody and refractory pouchitis. Dig Dis Sci 1995;40:1866-72.

9. Vecchi M, Gionchetti P, Bianchi MB, et al. p-ANCA and development of pouchitis in ulcerative colitis patients after proctocolectomy and ileoanal pouch anastomosis. Lancet 1994;344:886-7.
10. Esteve M, Mallolas J, Klassen J, et al. Antineutrophil cytoplasmic antibodies in sera from colectomised ulcerative colitis patients and its relation to the presence of pouchitis. Gut 1996;38:894-8.

11. Lennard-Jones JE. Classification of inflammatory bowel disease. Scand J Gastroenterol 1989;24(Suppl 170):2-6.

12. Sandborn WJ. Pouchitis following ileal pouch-anal anastomosis: definition, pathogenesis, and treatment. Gastroenterology 1994;107:1856-60.

13. Haagsma EB, Mulder AHL, Gouw ASH, et al. Neutrophil cytoplasmic autoantibodies after liver transplantation in patients with primary sclerosing cholangitis. J Hepatol 1993;19:8-14.

14. Zins BJ, Sandborn WJ, Penna CR, et al. Pouchitis disease course after orthotopic liver transplantation in patients with primary sclerosing cholangitis and an ileal pouch-anal anastomosis. Am J Gastroenterol 1995;90:2177-81.

15. Kartheuser AH, Dozois RR, Wiesner RH, LaRusso N-F, Ilstrup DM, Schleck CD. Complications and risk factors after ileal pouch-anal anastomosis with reservoir for ulcero-hemorrhagic rectocolitis associated with primary sclerosing cholangitis. Ann Chir 1993;47:1000-8.

16. Penna C, Dozois R, Tremaine W, et al. Pouchitis after ileal pouch-anal anastomosis for ulcerative colitis occurs with increased 
frequency in patients with associated primary sclerosing cholangitis. Gut 1996;38:234-9. 


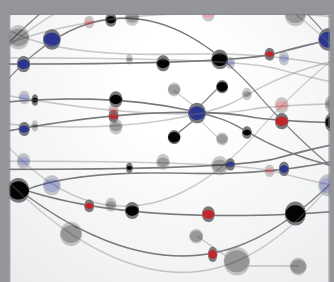

The Scientific World Journal
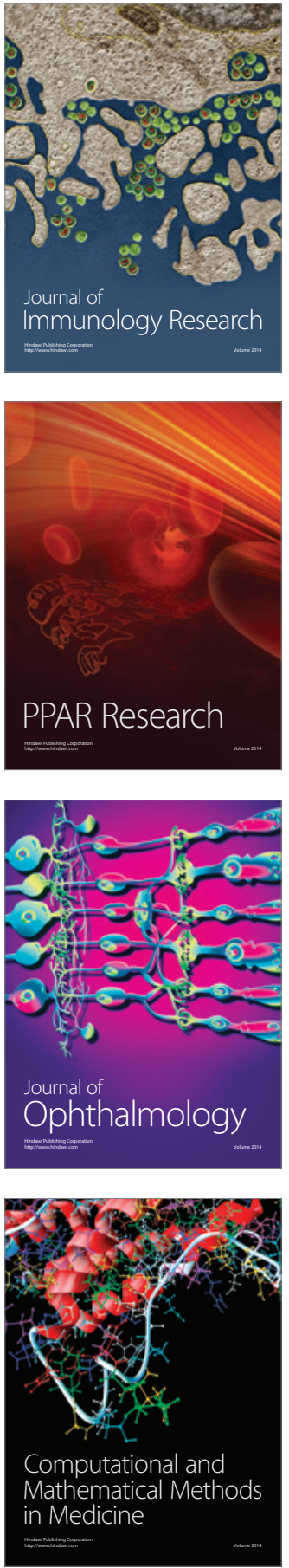

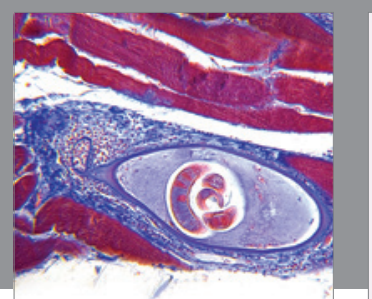

Gastroenterology Research and Practice

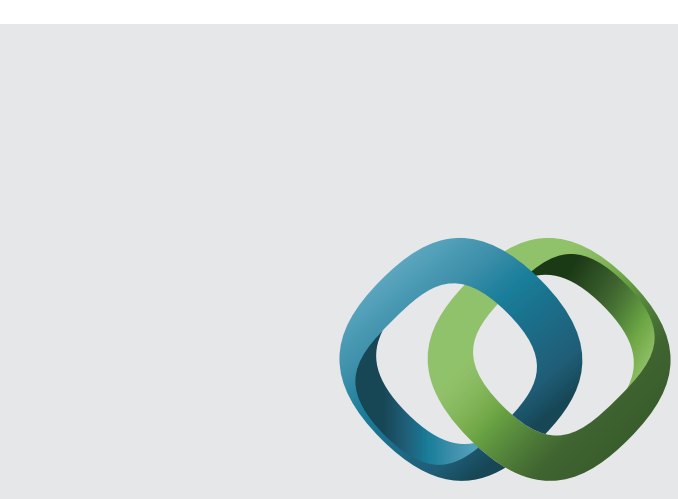

\section{Hindawi}

Submit your manuscripts at

http://www.hindawi.com
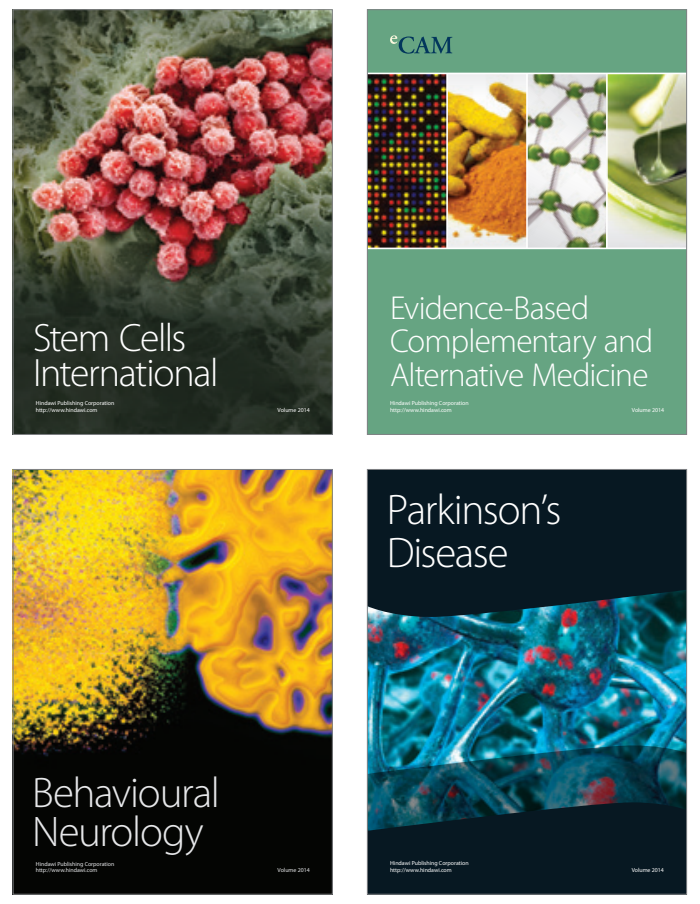
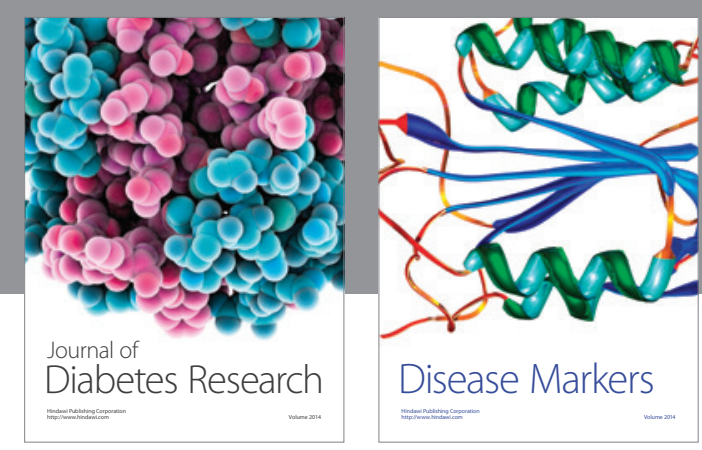

Disease Markers
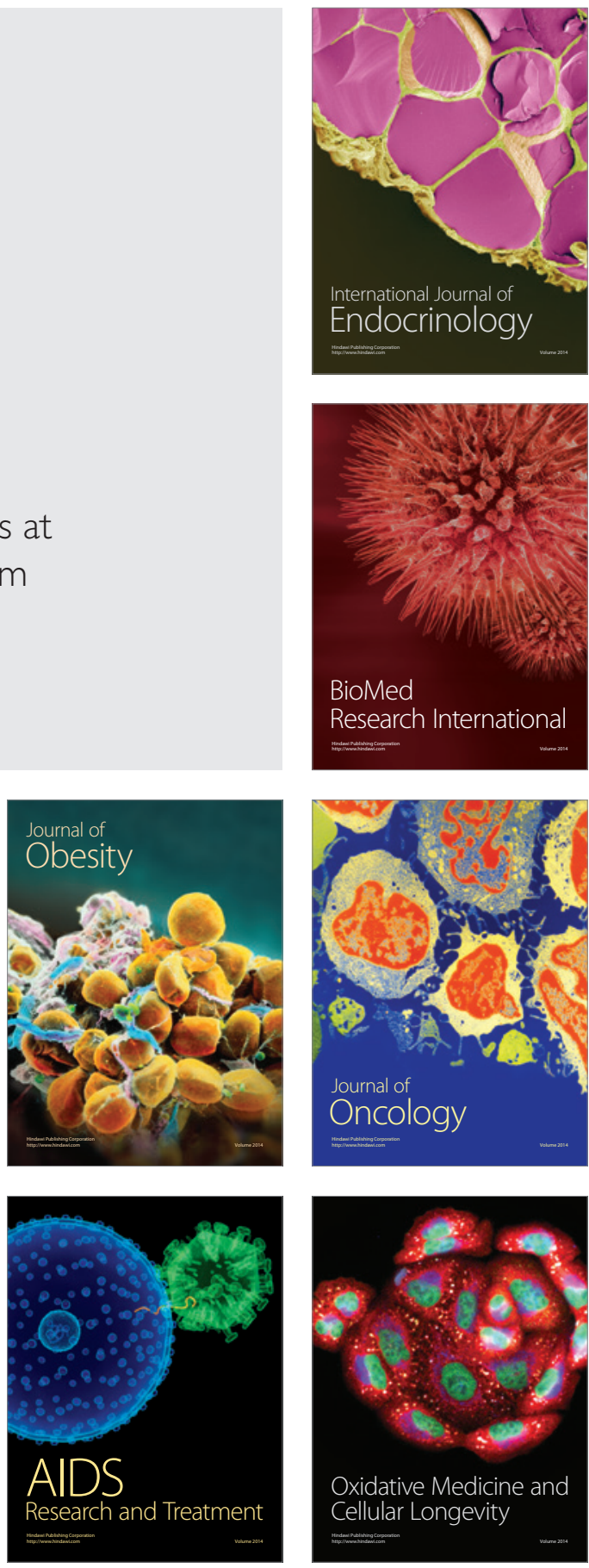